\title{
Application of Artificial Neural Networks to Predict the Carbon Content and the Grain Size for Carbon Steels
}

\author{
A. M. Abdelhay \\ Production Engineering Department \\ Faculty of Engineering, University of Helwan, Helwan, \\ Cairo 11792-Egypt. \\ Email : Abdelhay1953@yahoo.com
}

\begin{abstract}
Neural networks and generic algorithms are two branches of artificial intelligence that can provide many benefits in engineering applications. The artificial neural networks (ANN) technologies provide on-line capability to analyze many inputs and provide information to multiple outputs, and also, have the capability to learn or adapt to changing conditions. No doubt that the determination of either of the carbon content or the grain size of carbon steel is a time consuming process; which involves a quite tedious work. This paper examines the feasibility of using an integration system between some measured ultrasound parameters; from nondestructive test (NDT), and a pre - learned ANN to facilitate the determination of grain size and carbon content for the tested samples. The results showed that grain size and carbon content of carbon steels can be well predicted using a trained neural networks, with an acceptable degree of errors and great reliability.
\end{abstract}

Keywords: Neural networks, Carbon steel, Grain Size, Ultrasound Parameters, Nondestructive Testing (NDT). 


\section{Introduction:}

Grain size of the material is an important engineering characteristic; which influences its properties. Traditionally, optical metallography technique is used to evaluate the grain size of the materials. Such commonly used technique is quite a time consuming and feasible only at representative locations of the material. But, the new trend is to use non-destructive testing (NDT). This NDT is widely applied in many applications due to the fact that it will not impair the future usefulness of the test object.

In a previous work by the author [1], efforts had been made to use longitudinal normal incident ultrasonic waves as a non destructive evaluation (NDE) technique for the estimation of the average grain size of carbon steels. By using statistical methods of regression - type, the results showed a great dependency of some ultrasound parameters upon the grain size of the tested microstructures. Instead of using longitudinal ultrasonic, Rayleigh surface ultrasonic waves were extensively used [2-4] to measure surface/subsurface grain size in polycrystalline materials.

Neural networks have seen an explosion of interest over the last few years [5-9], and are being successfully applied across an extraordinary range of problem domains, in areas as diverse as finance, medicine, engineering, geology and physics. Indeed, anywhere that there are problems of prediction, classification or control, neural networks are being introduced. This sweeping success can be attributed to a few key factors. The first factor is that neural networks are very sophisticated modeling techniques capable of modeling extremely complex functions. In particular, neural networks are nonlinear. For many years linear modeling has been the commonly used technique in most modeling domains since linear models have well-known optimization strategies. Where the linear approximation was not valid, which was frequently the case, the models suffered accordingly. The second factor is that neural networks also keep in check the curse of dimensionality problem that bedevils attempts to model nonlinear functions with large numbers of variables. The third and final factor is the easiness of use, where neural networks can learn by example. The neural network user gathers representative data, and then invokes training algorithms to automatically learn the structure of the data. The biggest problem for the application of neural networks technique is the selection of its independent variables inputs, topology and the connection weights.

This works tries to demonstrate the great practical capability of artificial neural networks (ANN) to be used not only as a tool for grain size prediction, but also to evaluate the percentage carbon content of the tested samples. Data used for inputs of the ANN are nondestructively measured ultrasound 
parameters. This proposed technique can be a better practical alternative procedure for the statistical technique, previously used by the author [1].

\section{Theoretical Background Approach}

In an effort to determine which of independent variable are going to be used as the predictor inputs to the ANN topology, the following sub-section highlights these variables. Also, this section introduces some of the ANN main concepts.

\subsection{Ultrasound Predictors}

Consider the reflection and transmission of ultrasound stress wave along the interface boundary A-B as schematically shown in Fig. (1). Using a coupling medium at the interface; such as petroleum gel, produces an acoustic matching impedance [10], so a complete transmission of the incident ultrasound stress or pressure waves exists. Also, at the probe-specimen interface boundary, continuity assumption can be held true for the propagation of the ultrasound stresses waves.

Ultrasound stress ( or pressure) continuity states:

$$
\sigma_{i}+\sigma_{r}=\sigma_{t}
$$

where $\sigma_{\mathrm{i}}, \sigma_{\mathrm{r}}$, and $\sigma_{\mathrm{t}}$ are the incident, reflected, and transmitted ultrasound stresses respectively.

The frequency - dependent terms can be written as [10]:

$$
\sigma_{n}=P_{n} e^{j(\omega t-k x)} \quad(\mathrm{n}=\mathrm{i}, \mathrm{r}, \mathrm{t})
$$

where $P_{n}=$ the amplitude of the ultrasound stress wave $\sigma_{n}$,

$\omega=$ the stress wave circular frequency,

$\mathrm{k}=$ the wave number $(\mathrm{k}=[\omega / \mathrm{V}], \mathrm{V}$ is the propagation sound velocity),

$\mathrm{t}$ and $\mathrm{x}=$ the time and distance traveled by the ultrasound stress wave in material respectively. 


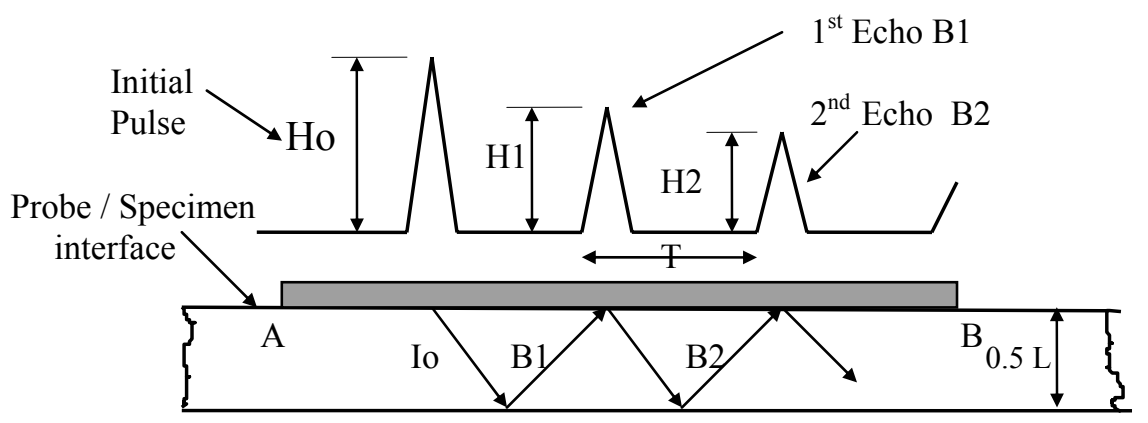

Fig. (1): Schematic diagram showing the pulse - echo signal, and the multiple reflection echoes from the back wall of a tested specimen.

The substitution of these terms; Eq. (2), into Eq. (1) yields

$$
P_{i}+P_{r}=P_{t}
$$

The reflection coefficient (R), can be defined in terms of the ultrasound stress wave amplitudes as:

$$
R=\frac{P_{r}}{P_{i}}
$$

The above equation is written as a ratio, since the absolute amplitudes $\mathrm{P}_{\mathrm{i}}$ and $\mathrm{P}_{\mathrm{r}}$ are of little interest in nondestructive evaluation (NDE). In the present work, the reflection coefficient (R), is going to be used as an index measure for the grain size of the tested specimens. As shown in Fig. (1), the amplitude $\left(\mathrm{P}_{\mathrm{n}}\right)$ of the ultrasound stress wave can be assumed to be proportional to the pulseecho heights (i.e $\mathrm{H}_{\mathrm{n}}$ ) ; which is measured in millivolt units.

Thus; Eq. (4) is going to be applied as:

$$
R_{1}=\frac{H_{1}}{H_{o}} \quad \text { and } \quad R_{2}=\frac{H_{2}}{H_{1}}
$$

The second quantitative predictor, which variables are going to be used in the prediction of the grain size of the tested specimens, is the attenuation coefficient $a_{n}$. This ultrasonic parameter $a_{n}$, is widely used to characterize material properties [3, 11-13], and is explained in more details in previous work [12]. 
Such attenuation coefficient is calculated as follows:

$$
a_{n}=\frac{20}{L} \log \left(\frac{H_{n}}{H_{n+1}}\right) \quad \mathrm{dB} / \mathrm{mm}
$$

where $\mathrm{H}_{n}$ and $\mathrm{H}_{n+1}$ are two consecutive amplitudes for the pulse - echo ultrasound wave signal- as shown in Fig. (1). While L, is the total traveled distance (i.e twice the thickness of test specimen) for the ultrasound stress wave.

Finally, a third predictor variable can be used for the indication of grain size (S) and the amount of carbon content (C), is the propagation ultrasonic velocity $(\mathrm{V})$; which is determined as the average velocity as follows:

$$
V=\frac{L}{T} \quad \mathrm{~m} / \mathrm{sec}
$$

where $\mathrm{T}$ (in sec units) is the time period between two consecutive echoes of the ultrasonic pulses, and $\mathrm{L}$ (in $\mathrm{m}$ units) as defined above.

\subsection{Neural Networks}

Artificial neural networks (ANNs) offer the potential to resolve a number of the problems encountered in different applied engineering fields. ANNs have been proposed as alternatives to the statistical analysis methods $[8,9]$.

An artificial neural network consists of a collection of processing elements that are highly interconnected and transform a set of inputs to a set of desired outputs. The result of the transformation is determined by the characteristics of the elements and the weights associated with the interconnections among them. By modifying the connections between the nodes the network is able to adapt to the desired outputs.

The neural network gains the experience initially by training the system to correctly identify pre-selected examples of the problem. The response of the neural network is reviewed and the configuration of the system is refined until the neural network's analysis of the training data reaches a satisfactory level. In addition to the initial training period, the neural network also gains experience over time as it conducts analyses on data related to the problem.

\subsubsection{Neural Network Description}

A simple network has a feedforward structure; where signals flow from inputs, forwards through many hidden units, eventually reaching the output 
units. A typical feedforward network is shown in Fig. (2), it has neurons (or nodes) arranged in a distinct layered topology. The input layer is not really neural at all: these units simply serve to introduce the values of the input variables. The hidden and output layer neurons are each connected to all of the units in the preceding layer. Again, it is possible to define networks that are partially-connected to only some units in the preceding layer; however, for most applications fully-connected networks are better.

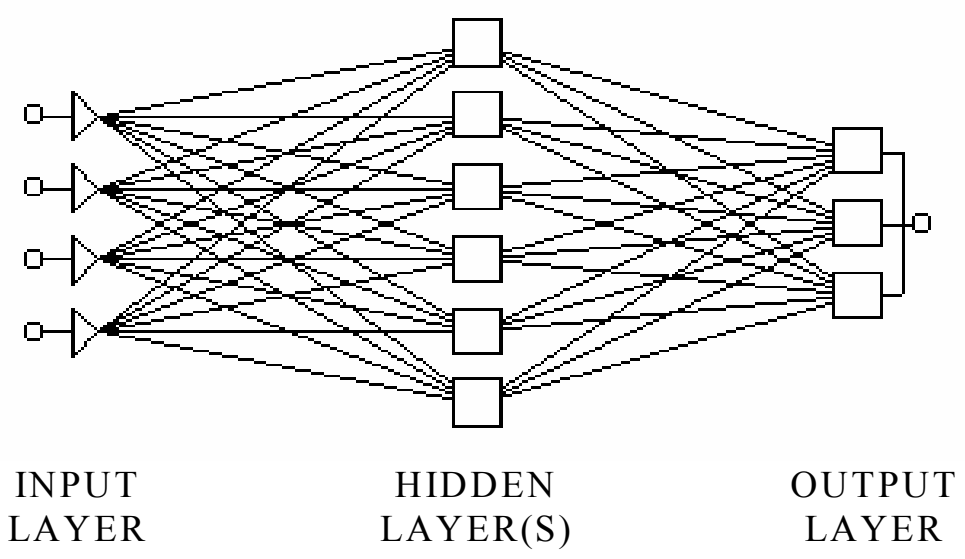

Fig. (2): Artificial Neural network (ANN) topology.

\section{Experimental Work}

\subsection{Test Samples}

Four different kinds of plain carbon steel (i.e. C15, C22, C35, and C45) were used in this work. Six samples were cut from each steel kind to a length of approximately $10 \mathrm{~mm}$. These round samples; with flat ends, were annealed. Range of annealing temperatures were from $850^{\circ} \mathrm{C}$ to $1050^{\circ} \mathrm{C}$ with $50^{\circ} \mathrm{C}$ increment. These different annealing temperatures were sufficient to impose different grain sizes.

Test specimens were then prepared for microstructure examination by optical microscope. From the micrographs, the average grain size was determined, by using the line intercept technique [14]. Thus a total of 24 different microstructures with different grain sizes were obtained (see Fig. 3b).

\subsection{Ultrasonic Evaluation}

A single $10 \mathrm{MHz}$ ultrasonic transducer (Krautkramer-Type) with a sensitivity diameter of $5 \mathrm{~mm}$ was mounted on each test sample as shown in Fig. 4. The ultrasonic waves from the transmission/reception (T/R) transducer were digitized and stored to a PC computer for later wave analysis by Wave Star (Ver 
2.1) package. For each acquired ultrasound wave, as the example shown in Fig. (3a), the first, second, and third echo amplitudes $\left(\mathrm{H}_{1}, \mathrm{H}_{2}\right.$, and $\left.\mathrm{H}_{3}\right)$, were determined. Also, the time period for the first two

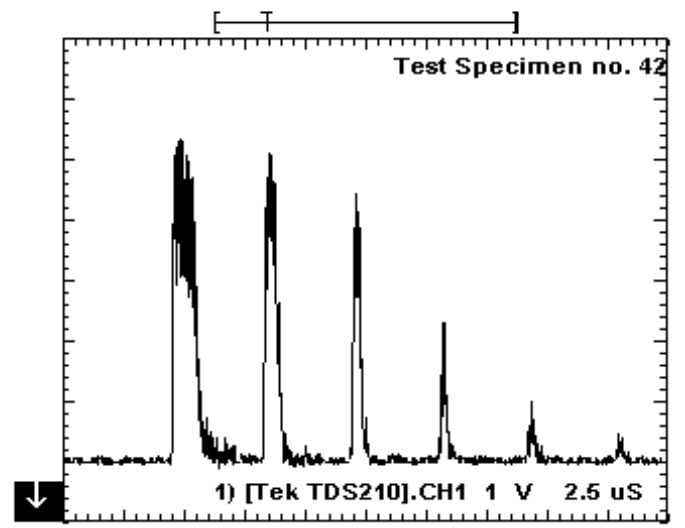

(a)

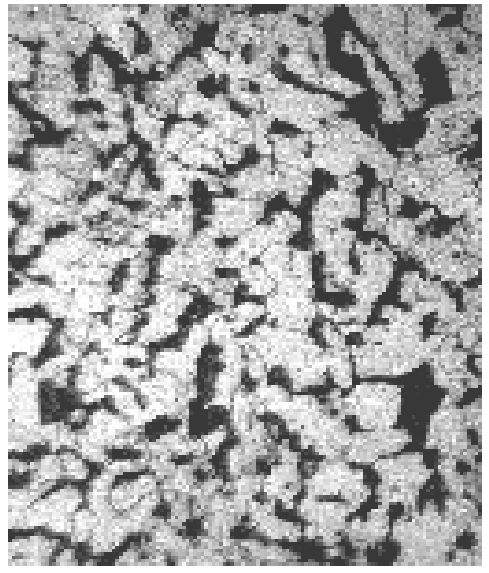

(b)

Fig. (3). A record (a) for the ultrasound wave image, and (b) its corresponding tested specimen microstructure (specimen no. 42).

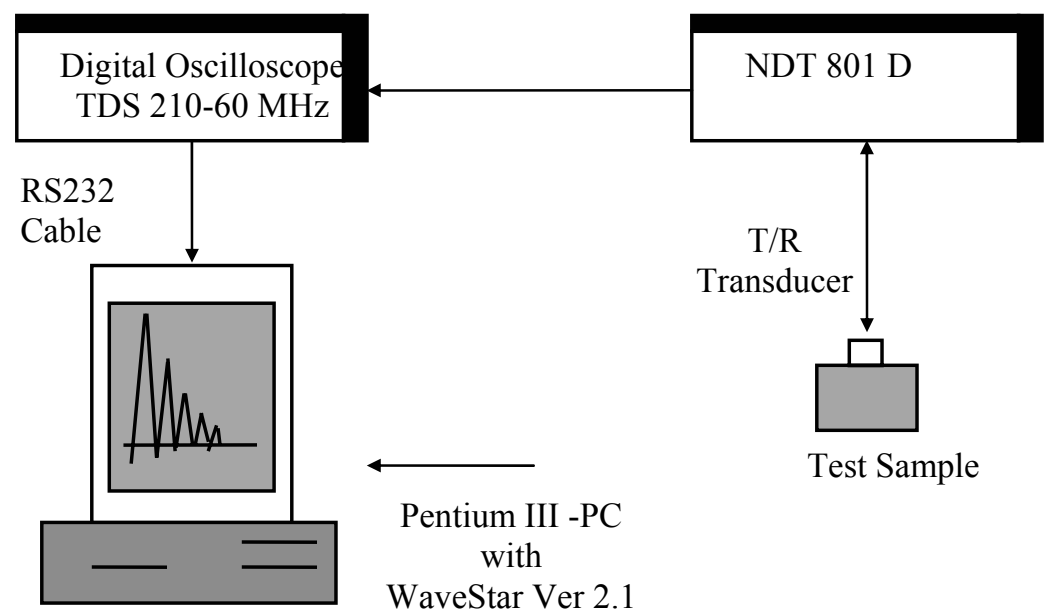

Fig. (4): Instrumentation used to measure ultrasonic waves parameters.

echoes was recorded. Using the average of three readings and Eqs. 5, 6, and 7; the five independent predictor variables; namely: the coefficient of reflections $\left(R_{1}\right.$ and $\left.R_{2}\right)$, the attenuation coefficients $\left(a_{1}, a_{2}\right)$, and the ultrasound velocity (V), can be defined for each test specimen, as shown in Table1. This data set was used as a training data, while an additional six readings were taken for some randomly selected test specimens to be used later as a validating data set. 


\section{Ann Implementation:}

Once, all the potential predictor variables (i.e. independents, inputs: $\mathrm{R}_{1}$ , $R_{2}, a_{1}, a_{2}$, and $V$ ), were numerically defined for each test sample, together with their known outputs of grain size (S), and carbon content $(\mathrm{C})$, the training of the neural network was ready to be carried out. Thus, the neural network had five input variables, and two

Table (1): Training and testing data

\begin{tabular}{|c|c|c|c|c|c|c|c|}
\hline \multirow{2}{*}{$\begin{array}{c}\text { Specimen } \\
\text { ID. }\end{array}$} & \multicolumn{5}{|c|}{ Ultrasound Measured Parameters } & \multicolumn{2}{|c|}{$\begin{array}{c}\text { Material } \\
\text { Characteristics }\end{array}$} \\
\hline & $a_{1}$ & $a_{2}$ & $\mathbf{R}_{1}$ & $\mathbf{R}_{\mathbf{2}}$ & $\mathbf{V}$ & C & $\mathbf{S}$ \\
\hline 111 & 0.09371 & 0.341312 & 0.818182 & 0.481481 & 5914.149 & 0.15 & 23 \\
\hline 121 & 0.026641 & 0.046407 & 0.932458 & 0.885312 & 5976.409 & 0.22 & 25 \\
\hline 131 & 0.041094 & 0.065784 & 0.901575 & 0.847162 & 5975.443 & 0.35 & 32 \\
\hline 141 & 0.068235 & 0.752114 & 0.853933 & 0.175439 & 6026.987 & 0.45 & 35 \\
\hline 211 & 0.098675 & 0.151621 & 0.807692 & 0.720238 & 5884.194 & 0.15 & 28 \\
\hline 221 & 0.008626 & 0.050591 & 0.981308 & 0.895238 & 6041.335 & 0.22 & 31 \\
\hline 231 & 0.030469 & 0.085067 & 0.934211 & 0.826962 & 5969.231 & 0.35 & 36 \\
\hline 241 & 0.056683 & 0.214119 & 0.878788 & 0.613793 & 5937.031 & 0.45 & 38 \\
\hline 311 & 0.000863 & 0.085174 & 0.998124 & 0.830827 & 6714.032 & 0.15 & 33 \\
\hline 321 & 0.019202 & 0.054775 & 0.962264 & 0.896078 & 6137.566 & 0.22 & 35 \\
\hline 331 & 0.027777 & 0.06224 & 0.942553 & 0.875847 & 5920.201 & 0.35 & 38 \\
\hline 341 & 0.031598 & 0.044687 & 0.930841 & 0.903614 & 6304.141 & 0.45 & 42 \\
\hline 411 & 0.071153 & 0.374354 & 0.871429 & 0.484778 & 6109.091 & 0.15 & 36 \\
\hline 421 & 0.133707 & 0.442017 & 0.716024 & 0.331445 & 5920.873 & 0.22 & 44 \\
\hline 431 & 0.218039 & 0.443423 & 0.616016 & 0.373333 & 5787.106 & 0.35 & 46 \\
\hline 441 & 0.070339 & 0.287329 & 0.851852 & 0.519451 & 6055.046 & 0.45 & 49 \\
\hline 511 & 0.264776 & 0.215093 & 0.565502 & 0.629344 & 5945.946 & 0.15 & 41 \\
\hline 521 & 0.126844 & 0.469549 & 0.746717 & 0.339196 & 5925.926 & 0.22 & 46 \\
\hline 531 & 0.195911 & 0.231549 & 0.631206 & 0.580524 & 5930.233 & 0.35 & 50 \\
\hline 541 & 0.058076 & 0.218947 & 0.881288 & 0.621005 & 6009.539 & 0.45 & 52 \\
\hline 611 & 0.056623 & 0.068231 & 0.882353 & 0.861021 & 5944.272 & 0.15 & 43 \\
\hline 621 & 0.299324 & 0.375477 & 0.534091 & 0.455319 & 6066.667 & 0.22 & 50 \\
\hline 631 & 0.257964 & 0.311091 & 0.61012 & 0.540084 & 5982.609 & 0.35 & 55 \\
\hline 641 & 0.382543 & 0.562704 & 0.421801 & 0.280899 & 6105.919 & 0.45 & 57 \\
\hline 13 & 0.032425 & 0.044013 & 0.921657 & 0.896039 & 6008.351 & 0.35 & 32 \\
\hline 22 & 0.031015 & 0.072558 & 0.934976 & 0.854815 & 6041.335 & 0.22 & 31 \\
\hline 33 & 0.037022 & 0.05821 & 0.924303 & 0.883589 & 5907.451 & 0.35 & 38 \\
\hline 44 & 0.125616 & 0.289903 & 0.765573 & 0.521234 & 6079.887 & 0.45 & 49 \\
\hline 51 & 0.102244 & 0.116817 & 0.825742 & 0.786162 & 5971.479 & 0.15 & 41 \\
\hline 64 & 0.17044 & 0.341366 & 0.421801 & 0.280899 & 6012.775 & 0.45 & 57 \\
\hline
\end{tabular}


dependent outputs. A commercially available ANN software called "Easy NN Ver 8.01" was used for the purpose of this work. This ANN software utilizes the multilayer perception (MLP) architecture shown in Fig. 2; which consists of one input layer, one output layer, and at least one hidden layer. A feedforward neural network architecture was selected based on the flexibility and applicability of the approach in a variety of problems [5 - 9]. The number of hidden layers, and the number of nodes in the hidden layers, can be manually selected or optimized by the ANN package. Each of the hidden nodes and the output node applied a Sigmoid transfer function of the form $(1 /(1+\exp (-\mathrm{x})))$ to the various connection weights.

Several ANN models were studied to examine the effects of reducing the number of predictor inputs, and changing the number of hidden layers upon the general prediction error of the proposed model. Table 2. Lists the different ANN models tested together with their configurations. In Table 2., the (x) and (-) indicate, respectively, whether the predictor at the top of the column is fed to the ANN model or not.

To evaluated the feasibility of each trained ANN model, the normalized root mean square error value (NSE) was used. Two NSE values were computed, one for the predicted value of the carbon content ( NSEC) and the second value for the grain size (NSES). The NSE is defined as follows:

$$
N S E=\sqrt{\frac{\sum\left(x-x_{o}\right)^{2}}{\sum x^{2}}}
$$

where $\mathrm{x}$ can be the carbon content $\mathrm{C}$ or the grain size $\mathrm{S}$, when computing the corresponding NSE value. When calculating the NSE, the value $\mathrm{x}_{\mathrm{o}}$ represents the predicted output value for the carbon content or the grain size.

Table (2): Different configuration for the tested ANN models.

\begin{tabular}{|c|c|c|c|c|c|c|}
\hline ANN & \multicolumn{5}{|c|}{ Active Input Combination } & ANN \\
\hline ID & $\mathbf{a}_{1}$ & $\mathbf{a}_{2}$ & $\mathbf{R}_{1}$ & $\mathbf{R}_{2}$ & $\mathbf{V}$ & Topology \\
\hline A & $\mathrm{x}$ & $\mathrm{x}$ & $\mathrm{x}$ & $\mathrm{x}$ & $\mathrm{x}$ & $5 \times 7 \times 2$ \\
\hline B & - & $\mathrm{x}$ & $\mathrm{x}$ & $\mathrm{x}$ & $\mathrm{x}$ & $4 \times 5 \times 2$ \\
\hline $\mathrm{C}$ & - & $\mathrm{x}$ & - & $\mathrm{x}$ & $\mathrm{x}$ & $3 \times 5 \times 2$ \\
\hline
\end{tabular}




\begin{tabular}{|c|c|c|c|c|c|c|}
\hline D & - & $\mathrm{x}$ & - & - & $\mathrm{x}$ & $2 \times 5 \times 2$ \\
\hline E & - & - & - & - & $\mathrm{x}$ & $1 \times 4 \times 2$ \\
\hline AA & $\mathrm{x}$ & $\mathrm{x}$ & $\mathrm{x}$ & $\mathrm{x}$ & $\mathrm{x}$ & $5 \times 7 \times 3 \times 2$ \\
\hline BB & - & $\mathrm{x}$ & $\mathrm{x}$ & $\mathrm{x}$ & $\mathrm{x}$ & $4 \times 5 \times 3 \times 2$ \\
\hline CC & - & $\mathrm{x}$ & - & $\mathrm{x}$ & $\mathrm{x}$ & $3 \times 5 \times 3 \times 2$ \\
\hline DD & - & $\mathrm{x}$ & - & - & $\mathrm{x}$ & $2 \times 5 \times 2 \times 2$ \\
\hline EE & - & - & - & - & $\mathrm{x}$ & $1 \times 4 \times 2 \times 2$ \\
\hline
\end{tabular}

\section{Results And Discussion:}

All proposed ANN models - listed in Table 2- were trained, using the 24 training examples of Table 1., for a target learning error of 0.04 , a learning rate of 0.6 , and with a learning momentum of 0.6 . Three of these ANN models (i.e. A, AA, and C) are shown in Fig. (5). The ANN model "A" shows a strong correlation between the ultrasound parameters in the following descending order:

$$
\mathrm{a}_{2} \Rightarrow \mathrm{R}_{2} \Rightarrow \mathrm{V} \Rightarrow \mathrm{R}_{1} \Rightarrow \mathrm{a}_{1} \Rightarrow \mathrm{R}_{1}
$$

This partially implies that the velocity of ultrasonic waves is strongly related to the amount of carbon content and to the grain size of the tested samples. Also, the ANN-model " $A$ " indicates that both of the second attenuation coefficient and coefficient of reflection (i.e. $a_{2}$ and $R_{2}$ ) are more sensitive to the predicted material characteristics than the first corresponding coefficients (i.e. $\mathrm{a}_{1}$ and $\mathrm{R}_{1}$ ). This observation can be attributed to that the second reflected echo is suffering more accumulated loss in ultrasound energy and absorption than the first ones; which in turn can be more valuable in the process of prediction of carbon content and grain size for the tested material. 


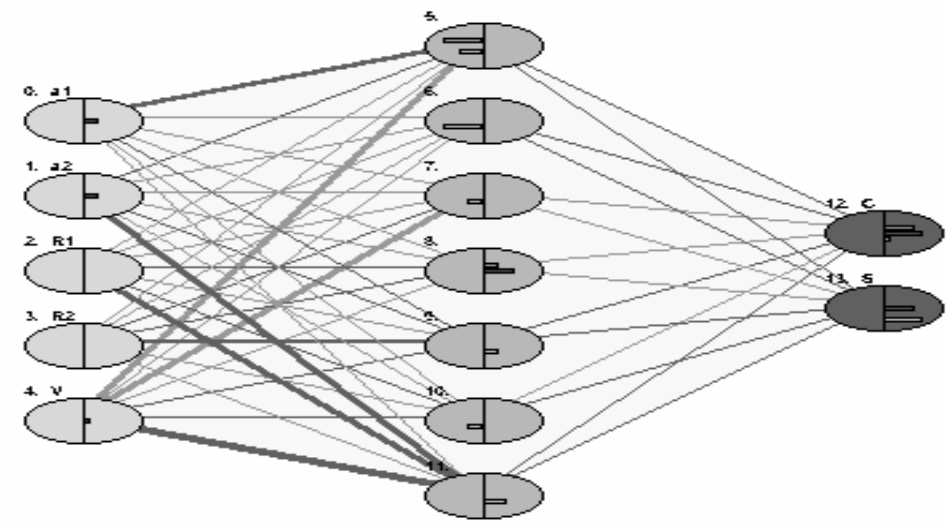

(a ) ANN model "A" - with [ $5 \times 7 \times 2]$ Topology.

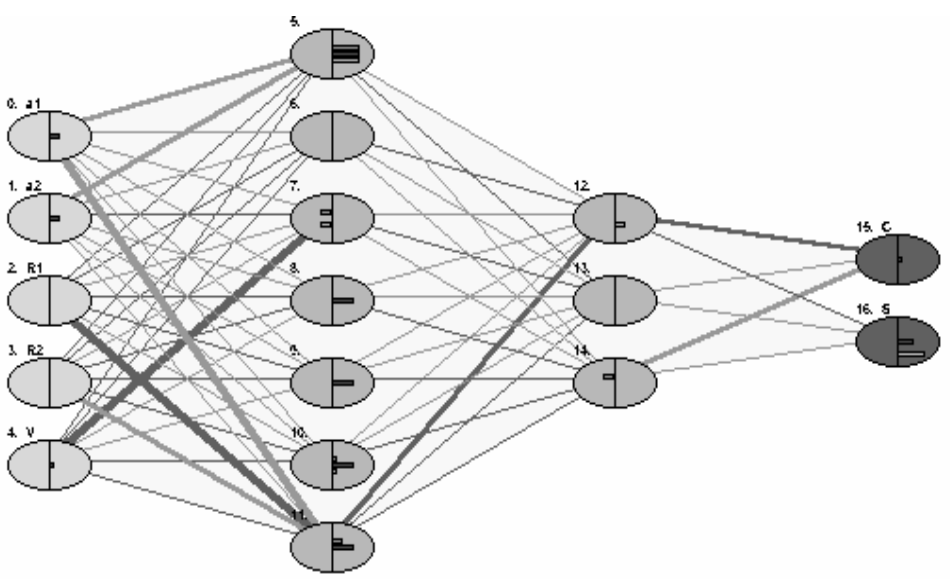

(b ) ANN model "AA" - with [ $5 \times 7 \times 3 \times 2$ ] Topology

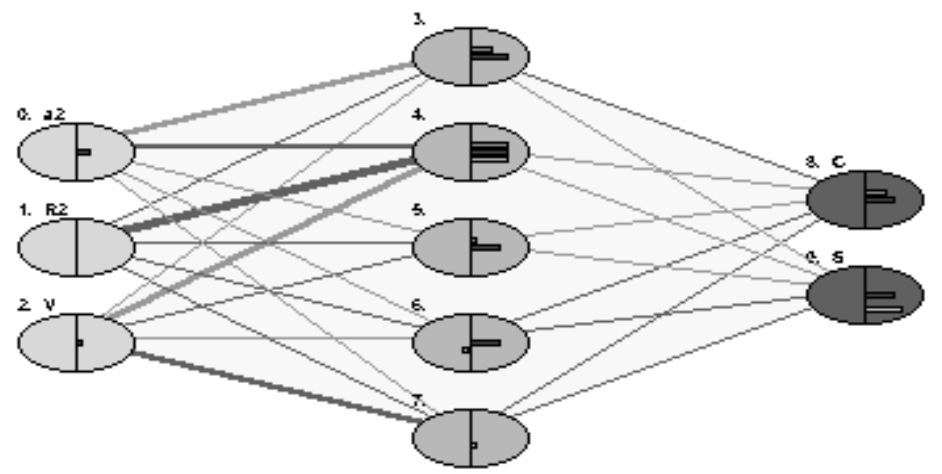

( c ) ANN model "C" - with [ 3 x 5 x 2 ] Topology

Fig. (5): Some examples for the proposed ANN -model configurations. 
In a trial to reduce the number of input predictors ( Notice that ANNmodel "A" uses 5 inputs), different ANN-models were trained using the same training data set of Table 1 . Once, the model was trained, the validating data set of Table 1 was introduced to the trained ANN - model, and predicted values for the carbon content and grain size were used in Eq. (8) to compute both of the normalized root mean square errors NSEC and NSES for the carbon content and the grain size respectively.

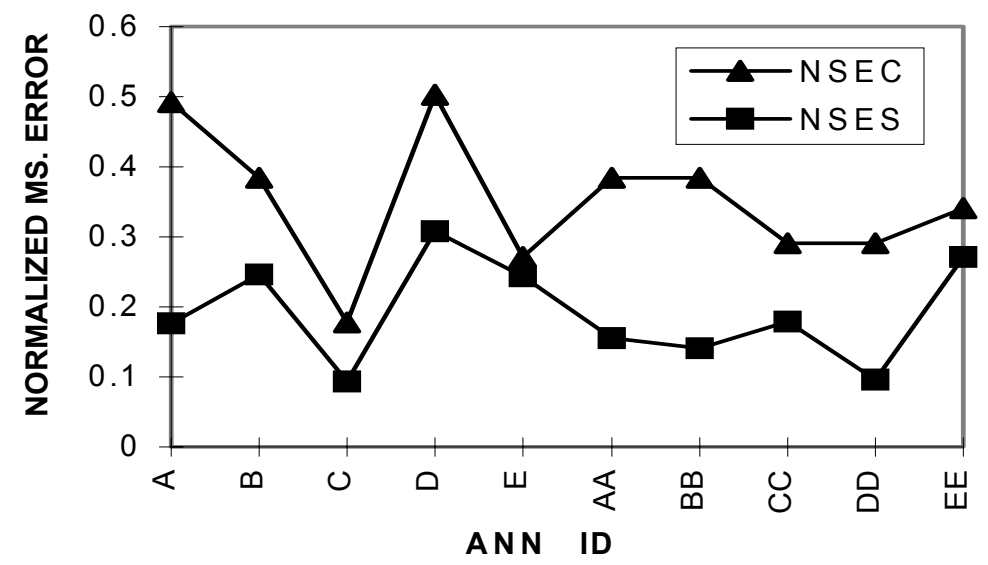

Fig. (6): Normalized error for different tested ANN models.

Results are shown in Fig. (6), and it can be seen that the "C"- ANN model is having the lowest overall values for the NSE. The topology of the "C" - model is shown in Fig. (5c)., it only uses the three most strongly related predictors $a_{2}->R_{2}->V$; which can be seen in Fig . (7). Such strongly related predictors can be sufficiently enough and mostly capable to be experimentally used in the prediction of carbon content and grain size of plain carbon steels with great success.

Individual measured values for both the carbon content and the grain size were compared with the prediction values of the "C" - ANN model, are shown in Fig. (8).

Computed relative prediction error for the carbon content was found to be 25 percent (Fig. 8a), while it was 2 to 19 percent for grain size (Fig. 8b). The reason that prediction error for carbon content was high; is that the values for such predicted output is not a continuous variable function (i.e $0.15 \Rightarrow 0.22 \Rightarrow$ $0.35 \Rightarrow 0.45$ stepwise values), so any deviation from the correct value will cause high error in the prediction of carbon content. 
3 of 3 Input columns in ascending order.

$\begin{array}{llrl}\text { Column } & \text { Input Name } & \text { Importance } & \text { Relative Importance } \\ 4 & \mathrm{~V} & 289.8191 & \\ 3 & \mathrm{R} 2 & 306.2857 & \\ 1 & \mathrm{a} 2 & 312.0310 & \end{array}$

Fig. (7): Relative importance of the predictor inputs of "C" - ANN model.
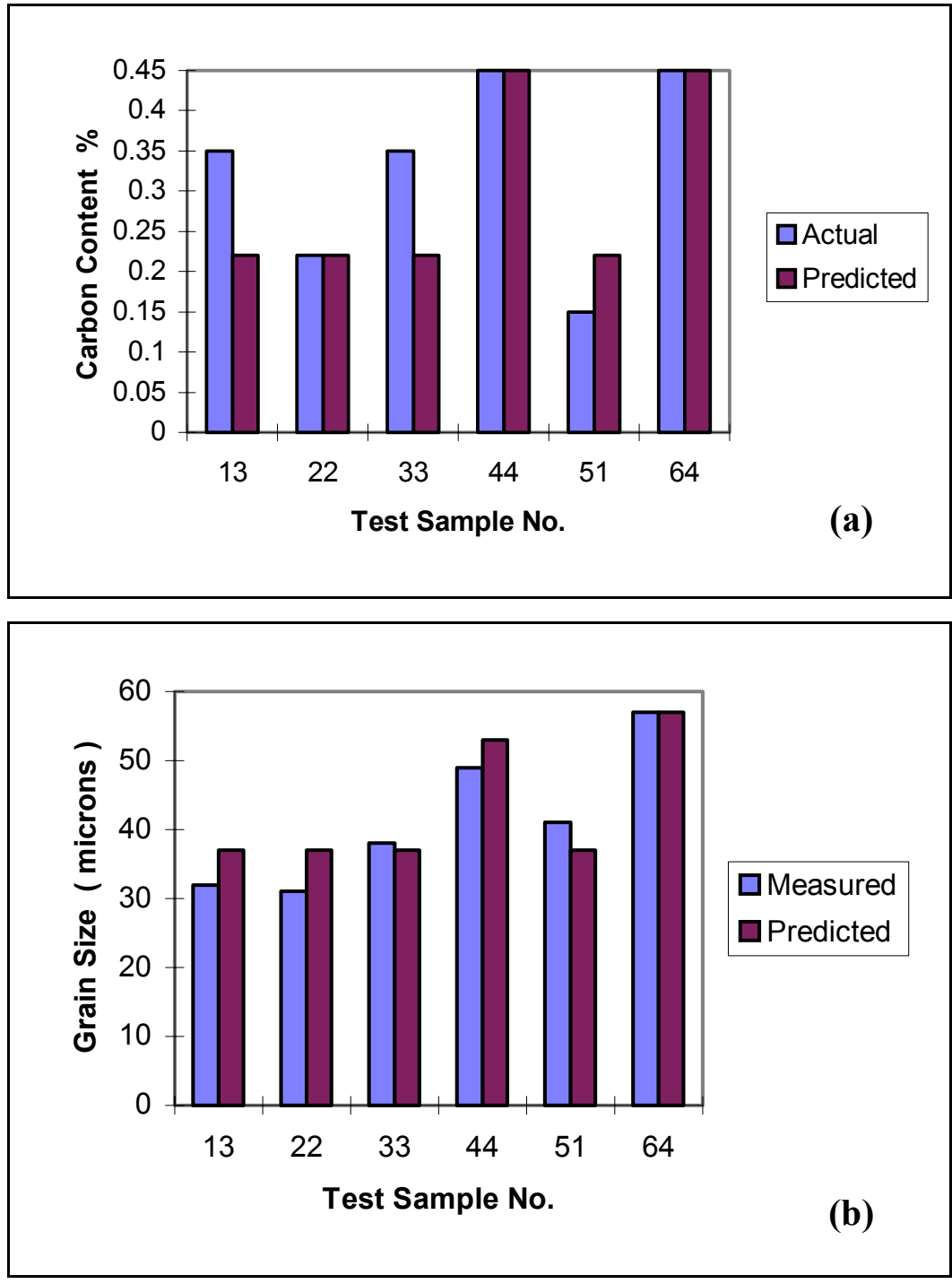

Fig. (8): Comparison between measured and predicted ANN values for carbon content and grain size for Plain carbon steel specimens, using the "C" - ANN model . 


\section{Conclusion:}

A technique was developed, which is applicable to the prediction of the average grain size and the carbon content of plain steel. The proposed technique is based on non-destructive evaluation procedure of using some selected ultrasound parameters, namely the coefficient of reflection $\left(\mathrm{R}_{1}, \mathrm{R}_{2}\right)$, the attenuation coefficient $\left(a_{1}, a_{2}\right)$, and the ultrasonic velocity $(V)$. As the ultrasound parameters were fed to an artificial neural networks, the best estimate values for the grain size and carbon content were predicted.

The most reached results were:

- The best proposed ANN - model, based on minimum normalized root square error was having a network of $3 \times 5 \times 2$ architecture,

- Three ultrasound parameters (i.e. $\mathrm{a}_{2}, \mathrm{R}_{2}$, and $\mathrm{V}$ ) were sufficient as predictor inputs for the proposed ANN model,

- Relative importance of the ANN model predictor in a descending order was: $a_{2}, R_{2}$, then $V$,

- Relative prediction error in the range of 2 to $19 \%$ was found in the prediction of grain size, and up to $25 \%$ for the prediction of carbon content, for the randomly selected specimens.

Thus, the proposed technique can be effective means of an integrated system of NDE and ANN; which may be extended to other engineering materials, and would be an interesting study to pursue.

\section{Acknowledgment:}

The author would like to thank his colleagues Dr. O. M. Dawood, Dr. M. I. Etman, Production Engineering department; Faculty of Engineering, Helwan University, for their objective discussions and supports. Also, my sincere thanks are due to Dr. A. El-Zomor ; Tabbin Institute for Metallurgical Studies (TIMS), for the assistance with the preparation of test specimens.

\section{References:}

1. Dawood O.M., Abdelhay A.M., El-Zomor, A., and El-Lethy, A.D., " $1^{\text {st }}$ Symposium on Heat Treatment of Metals and Alloys", TIMS Cairo, Egypt, pp. 123 -134, Nov. 3-5 (2001).

2. Palanichamy, P., and Jayakumar, "Trends in NDE Science and Technology", Proceedings of the $14^{\text {th }}$ World Conf. On NDT, New Delhi, Vol. 4, pp. 2253-2258, Dec. 8-13 (1996).

3. Wang. W., Rokhlin, S.I.Lippoid, J.C., and Adler,L., Material Evaluation, 47, 1388 (1989). 
4. Sladojevic, B., Trends in NDE Science and Technology, "Proceedings of the $14^{\text {th }}$ World Conf. On NDT", New Delhi, Vol 4, pp. 2605-2610, Dec. 8-13 (1996).

5. Rai, N; and Pitchumani, R AF., ASME, New York, USA, (1996).

6. Aboul-Nour, A.A., Shoukry, S.N., El-Sonbaty, I., and Ata, M.M., Int. Conf. PEDC, Alex. Univ., pp.427-435, Jan. (1997).

7. Altschuler, E., Gavarini, H., Perazzo, R. P. J., Pignotti, A., and Reich, S. L., Trends in NDE Science and Technology, Proceedings of the $14^{\text {th }}$ World Conf. On NDT, New Delhi, Vol. 4, pp. 1841- 1844, Dec. 8-13 (1996).

8. Etman, M. I., Dawood, O. M., and Abdelhay, A. M., PEDD6 , Ain Shams University, Cairo, Egypt, pp. 192 - 193, Feb. 12-14 (2002).

9. Azouzi, R; Guillot, M, of Machine Tools and Manufacture, 1201, 37(9), (1997).

10. Bray, D. E. and Stanley, R. K., " Nondestructive Evaluation- A tool in design, manufacturing and service", Mc Graw Hill Book Co., N.Y., (1989).

11. Ogilvy, J. A. and Culverwell, I. D., Ultrasonics, 29, 490 (1991).

12. Abdelhay, M.,A., and Mobark, I. M. I., PEDD6, Ain Shams University, Cairo, Egypt, pp. 607-617, Feb. 12-14 (2002).

13. Abdelhay, A. M. and Dawood O. M., $7^{\text {th }}$ Inter. Conf. On PEDAC, Alex, Egypt, Vol. III, pp:1431-1441, Feb. (2001).

14. Avner, S. H.," Introduction to Physical Metallurgy", $2^{\text {nd }}$ Ed.,Mc Graw Hill (1987). 